\title{
Single and Double Valence Configuration Interactions for recovering the exponential decay law while tunneling through a molecular wire
}

\author{
Mathilde Portais ${ }^{\text {a }}$ Mohamed Hliwa ${ }^{\mathrm{b}}$ Christian Joachim ${ }^{\text {a,c }}$ \\ ${ }^{a}$ CEMES-CNRS, 29 rue J. Marvig 31055 Toulouse Cedex4 France \\ ${ }^{\mathrm{b}}$ Faculté des Sciences Ben M'Sik, Université Hassan II de Casablanca, BP \\ 7955-Sidi Othman, Casablanca Morocco \\ ${ }^{\mathrm{c}}$ International Centre for Materials Nanoarchitectonics (MANA), National \\ Institute for Materials Science, 1-1, Namiki, Tsukuba, Ibaraki 305-0044, Japan
}

\begin{abstract}
The exponential decay of the electronic transmission through a molecular wire with its length is calculated using a Configuration Interaction Elastic Scattering Quantum Chemistry (CI-ESQC) theory [1,2]. In the HOMO-LUMO gap and in a one-electron approximation, this decays is exponential since the scattering matrix comes from a product of spatial propagators along the wire. In valence SD-CI description, such a product is not existing. An effective one was numerically obtained from the CI-ESQC scattering matrix. Fluctuations over the effective CI-exponential decay come from the truncation of the full CI basis set and also from many-body exchange-correlation effects along the molecular wire.
\end{abstract}

Key words: molecular wire, exponential decay, multi-configuration, tunnel junction, transmission coefficient

\section{Introduction}

For molecular electronics, the design of intramolecular electronics circuits depends on electronic circuit laws that remains to be mathematically consolidated [3]. For macroscopic electrical circuits, the mesh and node circuit laws were long established by G. Kirchhoff in the end of the 19th century. One well known consequence of those laws is that the overall resistance $\mathrm{R}$ of $\mathrm{N}$ identical resistance $r$ interconnected in series is just the linear sum $R=$ N.r. By the middle of the 20th century, it became clear that the overall transmission coefficient $\mathrm{T}$ for an electron tunneling through a series of $\mathrm{N}$ identical tunnel 
barriers of electronic transmission coefficient To is given by the power law $\mathrm{T}=$ $T o^{N}$ supposing no quantum phase loss along the single electron tunnel path. This leads to the well known mathematical expression $\mathrm{T}=e^{N \cdot \log (T o)}$.

Returning to the tunnel current intensity passing through a metal-moleculemetal tunnel junction, the above tunnel series law implies that the longer the molecular junction electron tunnelling path, the smaller the current intensity $[4,5]$. This exponential decay has indeed been observed experimentally on various molecular wires $[6,7,8,9,10,11,12,13,14,15,16,17,18]$. Its inverse decay length $\beta(E)$ is usually measured at low voltages ( $\mathrm{E}$ closed to the junction electrode Fermi energy Ef) and is ranging from about $1.0 \AA^{-1}$ [19] to the small $0.06 \AA^{-1}$ (value found for energies closed to a tunneling resonance [20]). This exponential decay is of a major importance since in many molecular electronic circuits, molecular devices are supposed to be interconnected using molecular wires $[22,23]$. Interconnecting a circuit with an exponentially current intensity decaying wire is not very promissing and requires the smallest possible $\beta(E)$ (see for example [21]).

Starting from a one-electron scattering approach, the low bias voltage energy dependent transmission coefficient $T(E)$ through a molecular wire made of a periodic repetition of $N_{l} \gg 1$ identical chemical groups (cells) can be analytically written $[4,5]$ :

$$
T(E)=T_{0}(E) e^{-\beta(E) l_{0} N_{l}}
$$

where the energy $\mathrm{E}$ of the incident electron scattered by the molecular wire junction ranges between the HOMO and the LUMO energies of the molecular wire in the so-called tunnelling transport regime [23]. $l_{0}$ is the effective length of a cell along the molecular wire and $T_{0}(E)$ the contact transmission coefficient. $T_{0}(E)$ does not depend on the molecular wire length but on its electronic coupling to the electrodes. $\beta(E)$ depends on the detail electronic structure of the cells and of their mutual interactions along the wire [21]. In this one electron approximation and in the middle of the molecular wire HOMO-LUMO energy gap near the Fermi level of the electrodes, $\beta\left(E_{f}\right)$ is given by [25]:

$$
\beta\left(E_{f}\right)=C \sqrt{\chi \frac{m^{*}}{m_{e}}}
$$

with $\chi$ the width of the HOMO-LUMO gap, $m^{*}$ the effective mass of a tunnelling electron in the junction, and $C$ a constant equal to $0.512 \mathrm{eV}^{-\frac{1}{2}} . \AA^{-1}$ [25].

In a monoelectronic picture, the periodicity of the molecular wire electronic structure intrinsically leads to the $T(E)$ exponential decay with length via the product law of the corresponding spatial propagators to calculate the one- 
electron scattering matrix [21,25]. This corresponds exactly to the $\mathrm{T}=T o^{N}$ series law mentionned above. Notice also that to quantify this decay with length, most of the theoretical studies have been carried out in a one electron approximation taking also into account in some studies many-body effects by perturbation [10].

The electronic scattering process through a molecular junction is intrinsically a many-body phenomenon where depending on all the resident electrons in the molecular junction and on their mutual interraction with the transfered electron, the tunneling decay is shapped. In this case, a product law for the spatial propagators doesn't come out analytically and the generalization of (1) cannot be found directly. One reason is the indiscernibility of electrons which forgives the separation of the spatial propagation of an incident charge from the configuration of all the other electrons resident in the molecular junction. Thus, the question arises on how mathematically an exponential decay emerges for $T(E)$ to exhibit an exponential decay in a multi-electronic description as compared with one-electron scattering description. By understanding this emergence, it may be possible to design better molecular wire with a small $\beta\left(E_{f}\right)$ by beneficiating for example from intramolecular correlation effects.

In this paper, we present a multi-configurational mathematical approach of the $T(E)$ exponential decay with the length of the molecular wire based on the CI-ESQC method [1,2]. Configuration Interaction CI-ESQC is the multiconfigurationnal generalisation of the mono-electronic ESQC (Electron Scattering Quantum Chemistry) method [26]. CI-ESQC allows to treat exactly the resident electrons along the molecular wire. In a first part, we briefly describe the CI-ESQC method and the electronic model for the molecular wire and the electrodes of the molecular junction. In a second part, the results of the CI-ESQC calculations for the $T(E)$ with different lengths of the molecular wire are presented before discussing these results and drawing conclusions on the nature of the tunnel conductance decay with the length of a regular in chemical structure molecular wire.

\section{The CI-ESQC calculation method}

To obtain the numerical results presented in this paper, the CI-ESQC method was used [1]. For a metal-molecule-metal junction with $m$ electrons, CI-ESQC allows to calculate the multi-configurationnal scattering matrix for the scattering process of a charge $q$ (an electron or a hole) on this $m$ electrons molecular junction. The calculation method is based on the general model presented figure 1. Considered as ballistic, the electrodes are modelled by 2 periodic semi-infinite wires of empty atomic orbitals. The molecule is modelled by $N$ molecular orbitals occupied by the $m$ electrons. In the present work, we are only interested by the scattering processes in where the tunnelling particle $q$ 


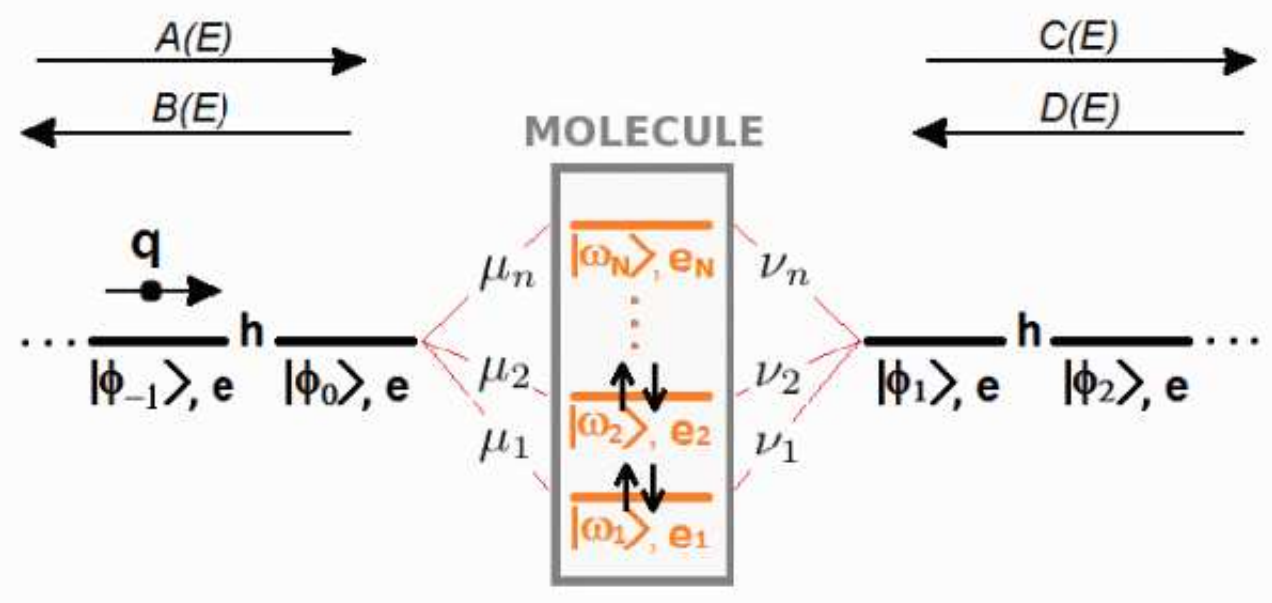

Figure 1. Model of the molecular junction used for the CI-ESQC calculations. The electrodes are empty except for the incident or the scattered particle $q$ (electron or hole). The molecule is represented by its molecular orbitals (MO) occupied by $m$ electrons not frozen in a particular electronic configuration during the scattering process.

(electron or hole) is scattered belongs to one electrode and is located far away from the molecular junction (e.g. case of weak molecule-electrode coupling). Two electron occupations of the molecular wire are considered, one in which the molecule takes an anionic form and the other in which it takes a cationic form. Those $(m+1)$ and $(m-1)$-electron occupation of the molecular wire are described in their ground states at the SD-CI (Single and Double Configuration Interaction) level. In this general treatment, the electron configurations made from the resident electrons and the transfered one are represented by a linear combination of Slater determinants associated to the ground state and to micro-states generated by electronic mono- and di-excitations from the ground state configuration. The resulting correlated ground sates of the $(m+1)$ and $(m-1)$-electron molecular wire are respectively used in the electron and hole sacattering calculations.Generalizing the one electron ESQC calculation method, the transmission coefficient is obtained for each kind of incident particle from the scattering matrix $\mathbf{s}(E)$ as $\left|\mathbf{s}(E)_{1,1}\right|^{2}$. For an incident hole, the transmission coefficient is noted as $T(E)=\left|t^{h}(E)\right|^{2}$, and for an incident electron: $T(E)=\left|t^{e}(E)\right|^{2}$. One can also calculate $T(E)$ for the case where hole and electron transmissions occur simultaneously, and in a coherent way: $T(E)=\left|t^{h}(E)+t^{e}(E)\right|^{2}$.

CI-ESQC was applied to the molecular wire junction presented in figure 2, with $N=30$ is the number of the orbitales of the molecular wire and $m=30$ 


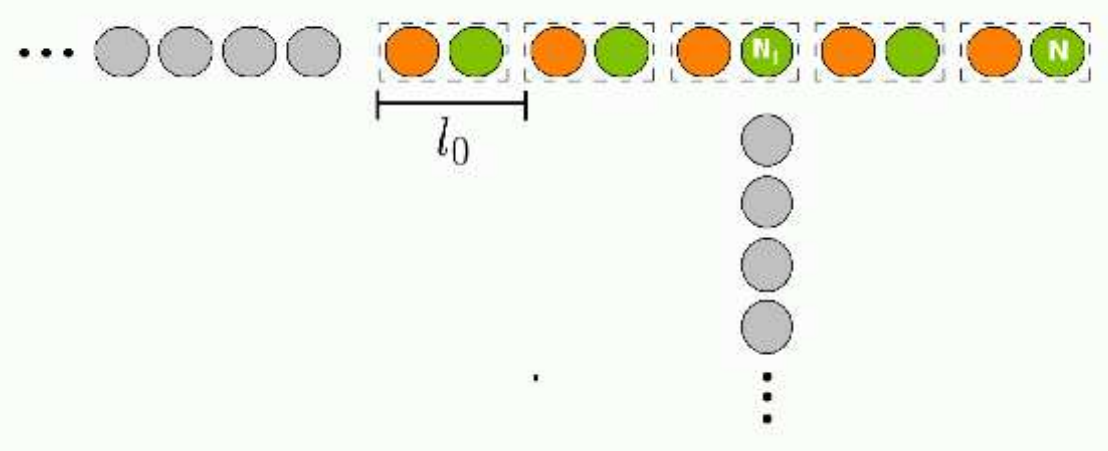

Figure 2. The spatial representation of the molecular wire considered in this letter and its electrodes forming the molecular junction. In this letter, this wire is made of 15 cells composed each of 2 quantum states with their corresponding atomic $\operatorname{orbital}(N=30)$. There are 30 electrons on this molecular wire plus the scattered particle $q$. The right perpendicular electrode is scanned along the molecular wire to calculate the multi-electronic transmission decay

. $l_{0}=3.0 \AA$.

is the number of the resident electrons. The molecular wire is made of $N / 2$ cells modelled each by a dihydrogen molecule and each hydrogen atom is represented by one 1s slater type orbital (STO), while the electrodes wires are described by a semi infinite chaine of hydrogen atoms. In this model system, 2-orbital/2-Hydrogen atoms were considered per cell to create an electronic gap between the HOMO and the LUMO of the so-constructed molecular wire. Describing the atom cell by a single STO is a rough approximation of the atomic orbitals normally used in quantum chemistry. But this approximation is sufficient to get qualitative results on the $T(E)$ decay with length. All the overlap and electronic integrals were analytically calculated starting from these orbitals [27]. For size consitancy and to keep the overall length $\frac{N l_{0}}{2}$ of the molecular wire constant, the configuration of the electrodes presented in figure 2 was used. Only the right electrode was slided along the wire to vary $\frac{N_{l} l_{0}}{2}$ i.e. the portion of the molecular wire connected between the electrodes of the molecular junction.

In the numerical calculations, it was not tractable to take into account all the Slater determinants constructed with $m+1=31$ particles distribued on 30 molecular orbitals (the Fig. 2 molecular wire) that is more than $2.10^{16}$ determinants. So, The valence CI-ESQC calculation was been restricted to only the 3 highest occupied and the 3 lowest unoccupied MOs. This lets to an CI active space made by 6 MOs and 6 electrons with free occupation status, the scattered particle can occupy any molecular orbital while the Pauli's principle is satisfied. Since the basis set is still too large within this truncated active 
space, only the single and double excited configurations besides the HF ground state state were considered. In the following we report, for comparison, also results of preliminar CI-ESQC calculations in which the $m$ resident electrons were ben frozen in the molecular wire ground state and in which the trasfered particle is free to scatter through any of the remaining $\frac{N}{2}$ virtual MOs of the molecular wire.

\section{The length dependent CI-ESQC calculated transmission coeffi- cient}

For the case in where the all $m$ resident electrons of the molecular wire are frozen, the calculated $\log [T(E)]$ spectra are reported in Figure 3 for $N_{l}=[10,12,14,16,18,20]$ with a cell length of $l_{0}=3.0 \AA$. Notice that this length controls for example the overlap between the atomic orbital of the molecular wire unit cells. In fig. 3a, the incident particle is an electron, in fig. $3 \mathrm{~b}$, the incident particle is a hole, and in fig. 3c, the junction is supposed to be in a coherent superposition of electron and hole scattering processes. As expected and in the energy ranges with no electron tunnelling resonances, the transmission coefficient is decreasing when $N_{l}$ increases. In a logarithmic scale, this decay is almost linear. A deviation only occurs at edges of the electrode energy band (left for a scattered electron and right for a scattered hole) because the complex valued band structure existing outside the propagative energy range of the electrode must be reached by continuity [25].

At low bias voltages, the energy range of interest for the tunnel transport regime is the electronic HOMO-LUMO gap of the molecular wire. Here and for the choosen value $l_{0}=3.0 \AA$, this electron energy gap is located between $E=-0.32 \mathrm{eV}$ and $E=2.28 \mathrm{eV}$. In this energy range and by using a linear regression between the calculated value of $\log (T(E))$ for the effective length $N l_{0}$ of the molecular wire, the $\beta(E)$ variation calculated for a junction in a coherent superposition of electron and hole scattering processes is reprted in the lower part of figure $4 . \beta(E)$ presents the same paraboloid variations as in a one electron ESQC calculations [25]. As shown in figure 4, The damping factor takes the values $\beta=0.0 \AA^{-1}$ at the edges of the electronic energy gap and reaches a maximum value of $\beta=0.37 \AA^{-1}$ near the Fermi level $E_{f}$ in the midddle the HOMO-LUMO energy gap. The upper part of figure 4 gives the standard deviation $\sigma$ between $\log [T(E)]$ and a linear function. Accordingly and as it is expected, the transmission coefficient $T\left(E_{f}\right)$ decays in average exponentially with length. The values of standard deviations are estimated 

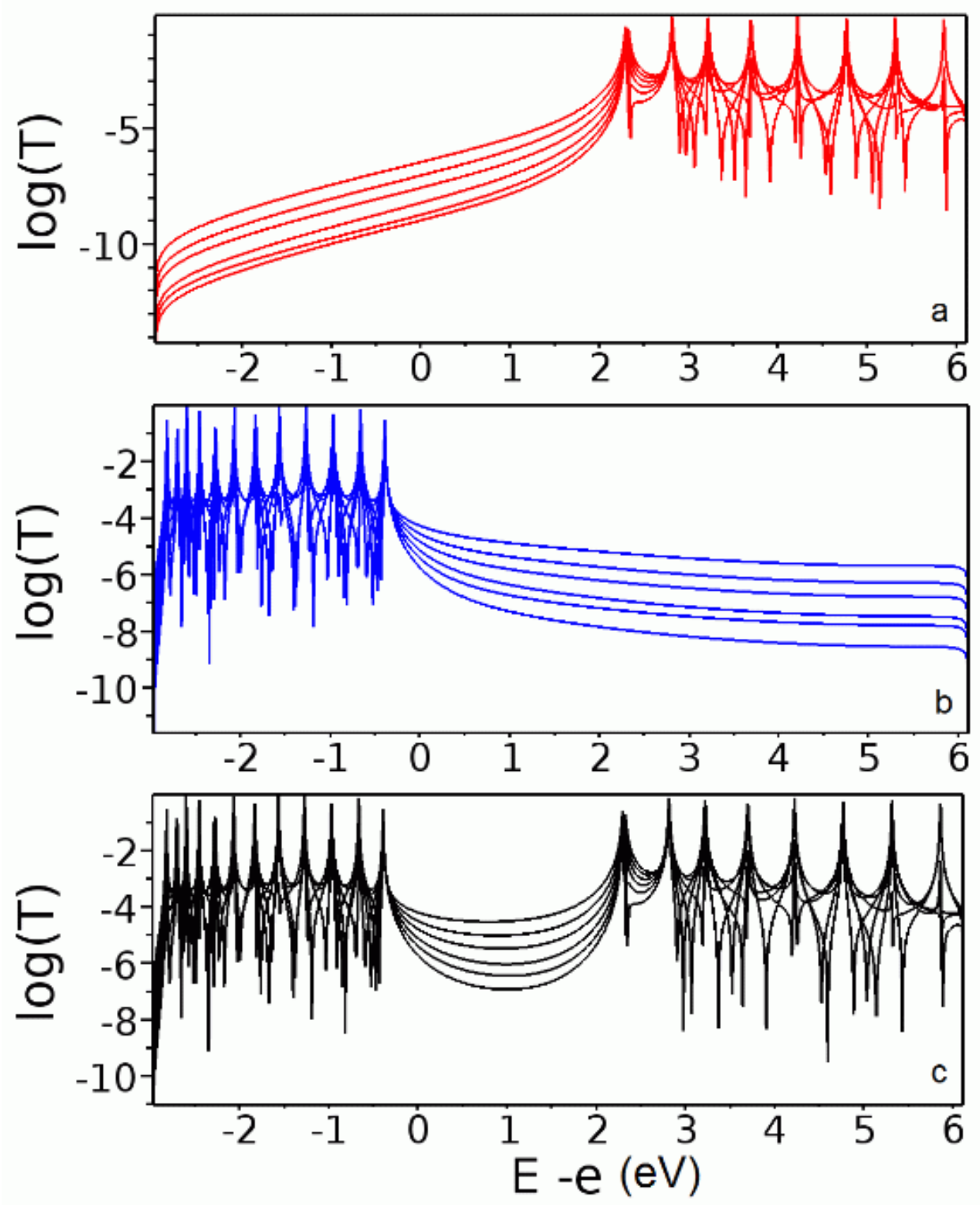

Figure 3. The $\mathrm{T}(\mathrm{E})$ transmission coefficients for different molecular wire lengths $N_{l}=[10,12,14,16,18,20]$ as a function of $E-e$ for the molecular junction figure 2 with $N=30$. Here, the 30 electrons of the molecular wire are frozen in their HF ground state. In (a), the scattered particle is an electron, in (b), a hole and in (c), the system is in a coherent superposition between an electron and a hole scattering states.

beings included in $10^{-1}$ and $10^{-2}$.

In figure 5 are reported the $\log [T(E)]$ spectra calculated by using CI-ESQC in the framework of an SD approximation. This is done for the same molecular wire using now an extended active space for describing the scattering process. As discussed above, the CI model space was restricted to Slater determinants generated from the ground state wave function by single and 

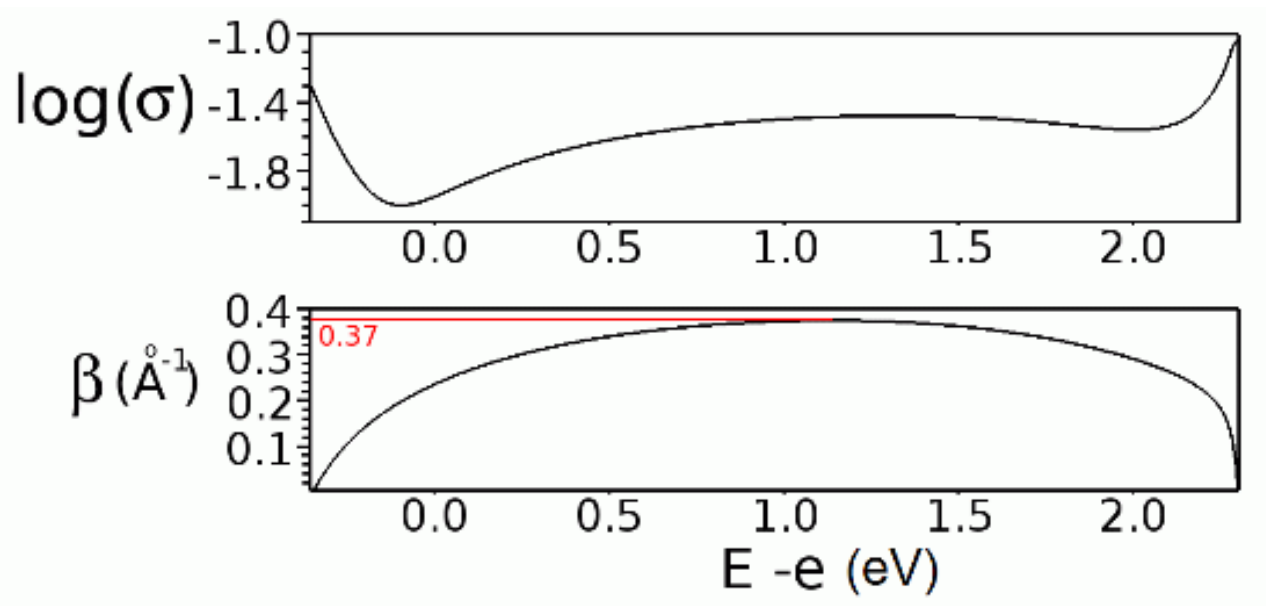

Figure 4. In the lower part, the decay parameter obtained by linear regression as a function of $E-e$ for $E$ in the electronic gap of the molecular wire figure 2. the 30 electrons of the molecular wire are frozen in their HF ground state. In the upper part, the standard deviation for each data set.

double-excitations (SD-CI). This is done in a zero order active space made by 6 valence frontier MOs and 6 electrons. The resulting transmission coefficienet spectra are reported in fig. $5 \mathrm{a}$ when the incident particle is an electron, in fig.5b when the incident particle is a hole, and in fig. $5 \mathrm{c}$ when the junction is in a coherent superposition of electron and hole scattering processes. Those 3 spectra are similar to the spectra obtained for the case for which the whole of the molecular wire resident electron are frozen (see figure 3 for comparison). This similarty can bee justified by the fact that the contribution of the HF ground state to SD-CI ground state is up to $99.8 \%$. For example, at $E=-0.32 \mathrm{eV}$, the tunnelling resonance peak corresponds to a multi-configurational state made up to $97.8 \%$ by the HF ground state and essentially completed by an other slater determinant wave functions associated to a mono-excitations made by annihiling one electron on the HOMO of the molecular wire and creating an electron on an atomic state of the atomic periodic chaine. In the same way, the peak at $E=2.24 \mathrm{eV}$ is resulting from state which is essentially a linear combination of the HF ground state (up to 98.9\%) and states with an excedent electron (promoted from the periodic chaine) on the LUMO of the molecular wire. Therefore, as consequence, the incident electron scattering process through the molecular wire is well reproduced when describing it by its HF frozen electrons approximation.

As shown in figure 6 and comparing with figure 4, the extended CI calculation induces a small effect on the energy variation of the tunnelling electron transmission damping factor $\beta(E)$. Its shape remained paraboloid and presents a 

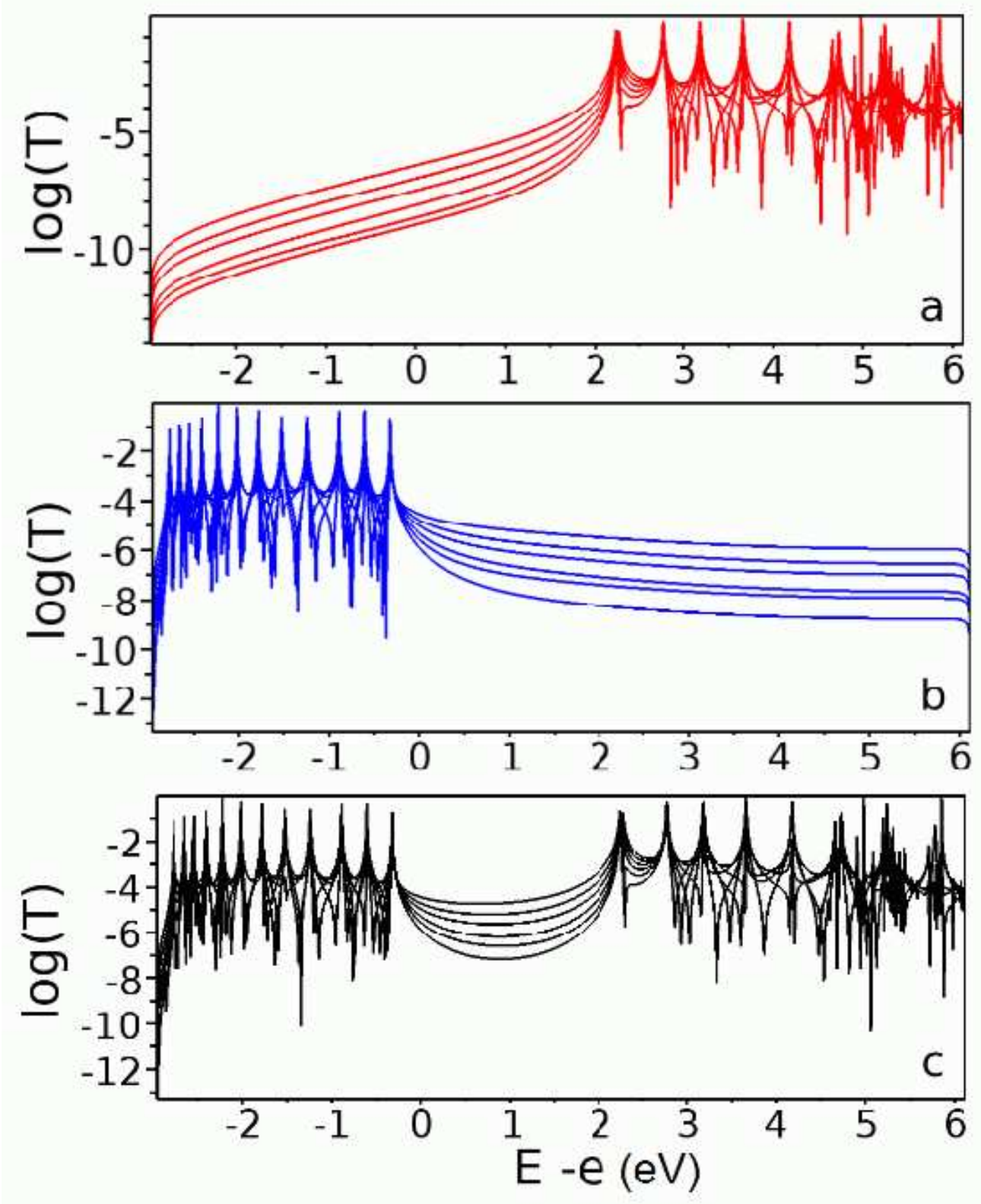

Figure 5. The $\mathrm{T}(\mathrm{E})$ transmission coefficients for different molecular wire lengths $N_{l}=[10,12,14,16,18,20]$ as a function of $E-e$ for the molecular junction figure 2 with $N=30$. Here, the active space is constructed starting from 6 MOs occupied by 6 electrons with the excitations limited to the mono and bi excitations. In (a), the scattered particle is an electron, in (b), a hole, and in c the system is in a coherent superposition between an electron and a hole scattering state.

maximum value equal to $\beta=0.38 \AA^{-1}$ instead the value of $\beta=0.37 \AA^{-1}$ obtained in the case where the whole of the resident electron is keepted frozen. By using equation (2) valid at the energy value $E=E_{f}$, the corresponding $\mathrm{m}^{*} / \mathrm{m}_{e}$ ratios are respectively equal to 0.22 and 0.20 . This increase of the electron effective mass $m^{*}$ is very small due to the low contribution of the excited 

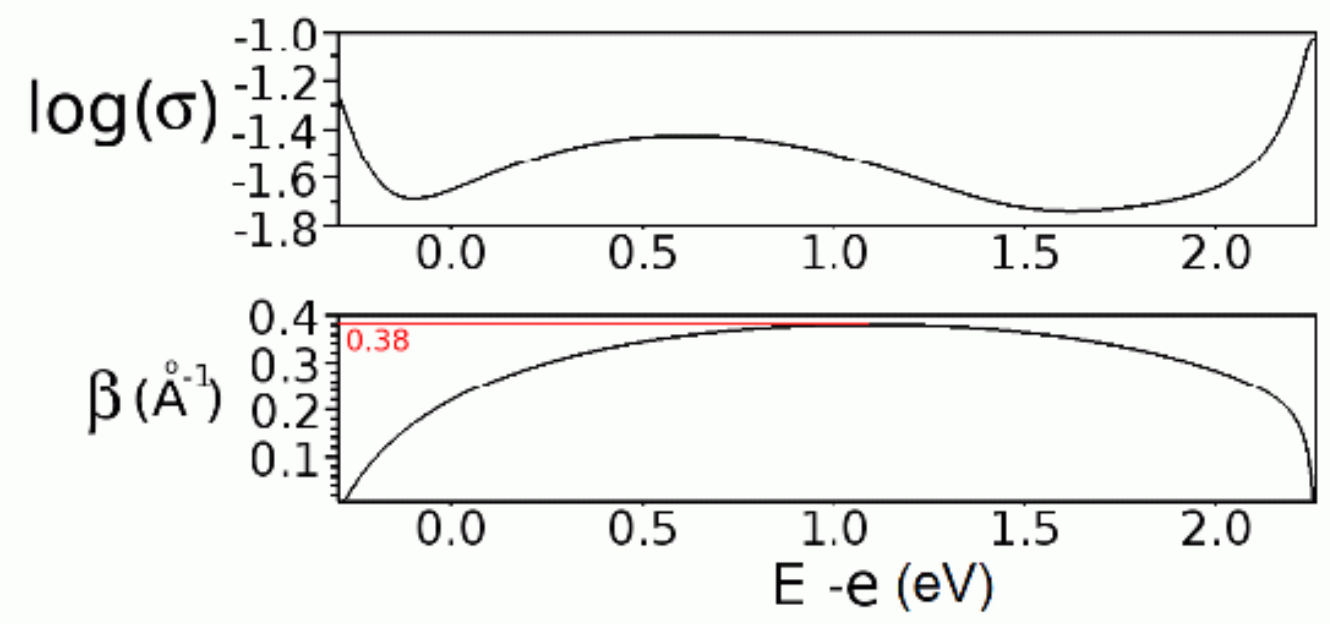

Figure 6 . In the lower part, the decay parameter obtained by linear regression as a function of $E-e$ for $E$ in the electronic HOMO-LUMO gap of the molecular wire figure 2. Here, the active space is constructed starting from 6 MOs occupied by 6 electrons with the excitations limited to the mono and bi excitations. In the upper part, the standard deviation for each data set.

states to the total wave function of the ground state. Its average standard deviation in the case of the valence SD-CI calculation is slightly greater than that in case of frozen electrons description, its minimum in the electronic gap being $10^{-1.74}$. It was reported elsewhere [28]; in framework of a mono-electronic approximation, the increase of the electron effective mass is a consequence of an increase of the spectral regidity of the electronic spectrum of the molecular wire. This observation can be generalized to the case of the present multielectronic description. Also, in the framework of the present SD-CI-ESQC calculation, the exponential decay of the tunnelling electron transmission coefficient $T\left(E=E_{f}\right)$ is similarly observed as in the case of o mono-electronic description.

\section{Discussion}

Valence SD-CI-ESQC multi-electronic calculations are demonstrating an average $T\left(E=E_{f}\right)$ exponential decay. In the one-electron approximation, such a decay is coming from the mathematical property of the spatial propagator to be decomposable along the molecular wire in a product of identical elementary spatial propagators, one per unit cell $[21,26]$. In the presented CI approach, such a mathematical decomposition is not tractable. To show how a product law is emerging at the CI-ESQC level, we have performed a numerical linear regression on the logarithm of the spatial propagator matrix $\mathcal{M}$ from site $\left|\phi_{n}\right\rangle$ 
to site $\left|\phi_{-n}\right\rangle$ on the electrodes for each type of incident particles. If a product law mathematically exists, the matrix $\mathcal{M}$ must have the form :

$$
\mathcal{M}=\mathrm{A}\left[\begin{array}{cc}
\lambda^{N_{l} / 2} & 0 \\
0 & \frac{1}{\lambda^{N_{l} / 2}}
\end{array}\right] \mathrm{B}
$$

In this case and for a given energy in the electronic gap, such a linear regression between $\frac{N_{l}}{2}$ and each matrix element of $\Re(\log (\mathcal{M})$ ) must lead (if existing) to the effective periodic components of the spatial propagator and deliver a new matrix whose eigenvalues are $|\lambda|$ and $\left|\frac{1}{\lambda}\right|$. For each type of scattered particle, we have found numerically that the $\Re(\log (\mathcal{M}))$ matrix elements vary almost linearly as a function of $\frac{N_{l}}{2}$. The standard deviation is between $10^{-1}$ and $10^{-2}$ in the HF frozen and in the non frozen cases respectively. For Valence SD-CIcalculation, at $E=1.5 \mathrm{eV}$ we find the value of $|\lambda|=1.69$, with a maximum standard deviation of 0.06 . Using the analytical expression of $\beta$ given in equation (2) when the spatial propagator matrix satisfies the expression (3), we

calculate the transmission damping factor to be $\beta=\frac{2 \log (|\operatorname{lambda}|)}{l_{0}}=0.353 \AA^{-1}$, this value of $\beta$ is equal to the value obtained from a direct linear regression of $T(E=1.5 \mathrm{eV})$.

Numerically, it is therefore possible to built up an effective product law along the molecular wire for the spatial propagator from site $\left|\phi_{n}\right\rangle$ to site $\left|\phi_{-n}\right\rangle$ of the electrodes and for each type of scattered particle. This explains why $T(E)$ decays also exponentially with length for the CI-ESQC calculations presented here even if there are non negligible fluctuations around this average exponential decay. Those fluctuations are certainly due to the drastic basis set truncation used in the present work. This truncation may not be fully adapted to the effective description of a tunnelling process. This opens the question of determining the best electronic active space to describe an electronic tunnelling process through a molecular junction. Calculations with longer wires and large basis are now required to verify the truncation effects. The fluctuations around the exponential decay may also correspond to a physical reality not yet accessible to experimental measurements.

\section{Conclusion}

The transmission coefficient through a molecular wire tunnel junction is well know experimentally to decay exponentially with the length of the molecular wire. This phenomenon can be demonstrated mathematically in a one electron approximation of this scattering process. In a many-body picture, it is 
not possible to analytically demonstrate such an exponential decay. Using our new CI-ESQC calculation technique, we have shown here that in tunnelling regime, the transmission coefficient through a molecular wire decreases exponentially on average with the length of this molecular wire. There are some fluctuations in energy around this average, which are occurring when the exchange interactions are taken into account in the CI frozen resident electrons approximation. Those fluctuations increase when the electron correlation effects are added via the valence SD-CI description. No exact analytic product law appears in the expression of the spatial propagator matrix of the scattering process. A detailed numerical study of this spatial propagator matrix shows that an effective product law can be extracted numerically. This explains the average exponential decay of the transmission coefficient with the length of the molecular wire. The fluctuations around this average exponential decay can be explained in two ways. They come mathematically from the truncation of Slater determinants basis set used pointing out the necessity to build up an active model space for tunnelling. They can also correspond to a physical reality, not observable for now because of the experimental precision. When tunnelling through a molecular wire on the long range, the effective exponential decay law numerically demonstrated here is a good indication that an electron (a hole) is only sensible to the average electronic structure found during its individual transfer path through a molecular wire.

\section{References}

[1] M. Portais and C. Joachim. "Electronic transmission through a single impurity in a multi-configuration scattering matrix approach" ; in Imaging and Manipulating Molecular Orbitals, pages 137 ̃̂â157.Springer, 2013.

[2] M. Portais and C. Joachim. "Hole-electron quantum tunnelling interferences through a molecular junction" ; Chem. Phys. Lett. 592 (2014) 272.

[3] C. Joachim. "Molecular electronics : Probing intramolecular circui laws" ; Nature Nano. 7 (2012) 620.

[4] V Mujica, M Kemp, and M.A. Ratner. "Electron conduction in molecular wires, application to scanning tunneling microscopy" ; J. Chem. Phys., 101 (1994) 6856.

[5] C. Joachim and J.F. Vinuesa. "Length dependence of the electronic transparency (conductance) of a molecular wire ; Europhys. Lett. 33 (1996) 635.

[6] L. Lafferentz, F. Ample, H. Yu, S. Hecht, C. Joachim, and L. Grill. "Conductance of a single conjugated polymer as a continuous function of its length" ; Science $\mathbf{3 2 3}$ (2009) 1193.

[7] V. Langlais, R.R. Schlittler, H. Tang, A. Gourdon, C. Joachim, and J.K. Gimzewski. "Spatially resolved tunneling along a molecular wire" ; Phys. Rev. Lett. 83 (1999) 2809. 
[8] D.J. Wold, R. Haag, M.A. Rampi, and C.D. Frisbie. "Distance dependence of electron tunneling through self-assembled monolayers measured by conducting probe atomic force microscopy, Unsaturated versus saturated molecular junctions" ; J. Phys. Chem B 106 (2002).

[9] M. Koch, F. Ample, C. Joachim, and L. Grill. "Voltage-dependent conductance of a single graphene nanoribbon" ; Nature nanotechnology 7 (2012) 713.

[10] A. Ferretti, G. Mallia, L. Martin-Samos, G. Bussi, A. Ruini, B. Montanari, and N.M. Harrison. "Ab initio complex band structure of conjugated polymers: Effects of hydrid density functional theory and GW schemes" ; Phys. Rev. B 85 (2012) 235105.

[11] W. Wang, T. Lee, and M.A. Reed. "Mechanism of electron conduction in selfassembled alkanethiol monolayer devices" ; Phys. Rev. B 68 (2003) 035416.

[12] S.H. Choi, B. Kim, and C.D. Frisbie. "Electrical resistance of long conjugated molecular wires" ; Science 320 (2008) 1482.

[13] E. Leary, H. Van Zalinge, S.J. Higgins, D. Bethell and D.J. Schiffrin. "Measurement of single molecule conductivity using the spontaneous formation of molecular wires" ; Phys. Chem. Chem. Phys. 6 (2004) 4330.

[14] M.U. Winters, E. Dahlstedt, H.E. Blades, C.J. Wilson, M.J. Frampton, H.L. Anderson, and B. Albinsson. "Probing the efficiency of electron transfer through porphyrin-based molecular wires" ; J. Am. Chem. Soc. 129 (2007) 4291.

[15] G. Sedghi, K. Sawada, L.J. Esdaile, M. Hoffman, H.L. Anderson, D. Bethell, W. Haiss, S.J. Higgins, and R.J. Nichols. "Single molecule conductance of porphyrin wires with ultralow attenuation" ; J.Am. Chem. Soc. 130 (2008) 8582.

[16] H. Sakaguchi, A. Hirai, F. Iwata, A. Sasaki, T. Nagamura, E. Kawata, and S. Nakabayashi. "Determination of performance on tunnel conduction through molecular wire using a conductive atomic force microscope" ; Appl. Phys. Lett. 79 (2001) 3708 .

[17] L. Luo and C.D. Frisbie. "Length-dependent conductance of conjugated molecular wires synthesized by stepwise click chemistry" ; J. Am. Chem. Soc. 132 (2010) 8854.

[18] G. Peng, M. Strange, K.S. Thygesen, and M. Mavrikakis. "Conductance of conjugated molecular wires, length dependence, anchoring groups and band alignment" ; J. Phys. Chem. C 113 (2009) 20967.

[19] L.A. Bumm, J.J. Arnold, T.D. Dunbar, D.L. Allara, and P.S. Weiss. "Electron transfer through organic molecules" ; J. Phys. Chem. B 103 (1999) 8122.

[20] C. Wang, A.S. Batsanov, M.R. Bryce, S. Martin, R.J. Nichols, S.J. Higgins, V.M. Garcia-Suarez, and C.J. Lambert. "Oligoyne single molecule wires" ; J. Am. Chem. Soc. 131 (2009) 15647.

[21] M. Magoga and C. Joachim. "Minimal attenuation for tunneling through a molecular wire" ; Phys. Rev. B 57 (1998) 1820. 
[22] C. Joachim, J.K. Gimzewski and A. Aviram. "Electronics using hybrid-molecular and mono-molecular devices" ; Nature 408 (2000) 541.

[23] C. Joachim, N. Renaud, and M. Hliwa. "The different designs of molecule logic gates" ; Advanced Materials 24 (2012) 312.

[24] C. Joachim and M.A. Ratner. "Molecular electronics, some views on transport junctions and beyond" ; Proc. Nat. Acad. Sci. USA 102 (2005) 8801.

[25] C. Joachim and M. Magoga. "The effective mass of an electron when tunneling through a molecular wire" ; Chem. phys. 281 (2002) 347.

[26] P. Sautet and C. Joachim. "Electronic transmission coefficient for the single-impurity problem in the scattering-matrix approach" ; Phys. Rev. B 38 (1988) 12238.

[27] A. Szabo and N.S. Ostlund. "Modern quantum chemistry, introduction to advanced electronic structure theory" ; Dover Publications. com. (1989).

[28] A. Lahmidi and C. Joachim. "Decay of the molecular wire conductance with length, the role of spectral rigidity" ; Chem. Phys. Lett. 381 (2003) 335. 